\title{
Ultrastructural and Metabolic Profiles on Single Muscle Fibers of Different Types after Hindlimb Suspension in Rats
}

\author{
Hiroaki TAKEKURA* and Toshitada YoshioKA** \\ * Department of Physiology and Biomechanics, National Institute \\ of Fitness and Sports, Kanoya, 891-23 Japan \\ ** Department of Physiology, St. Marianna University School of Medicine, \\ Miyamae-ku, Kawasaki, 213 Japan
}

\begin{abstract}
The relationships between ultrastructural and metabolic profiles in different types of single muscle fiber after hindlimb suspension in rats were examined. Glycolytic (lactate dehydrogenase, LDH; phosphofructokinase, PFK) and oxidative (succinate dehydrogenase, $\mathrm{SDH}$; malate dehydrogenase, $\mathrm{MDH}$ ) enzyme activities in extensor digitorum longus (EDL) and soleus (SOL) muscles were measured. Relative mitochondrial and lipid droplet volumes were also measured in single muscle fiber of different types. Glycolytic enzyme activity in EDL muscle and oxidative enzyme activity in soleus muscle decreased following suspension for 2 weeks. LDH and PFK activities in fast-twitch (FG, fast-twitch glycolytic; FOG, fast-twitch oxidative glycolytic) fibers and oxidative enzymes in FOG and FG fibers decreased following suspension. Relative mitochondrial volume decreased significantly in all types (SO, slow-twitch oxidative; FOG, and FG) of fibers following suspension. The mitochondrial volume in SO fiber of the control group was significantly $(p<0.01)$ higher than that of suspended group; however, SDH and MDH activities were not different between the control and suspended groups. The structural and metabolic changes following hindlimb suspension were influenced by different factors, respectively. Changes in ultrastructural and metabolic profiles in response to the hindlimb suspension differed according to the type of fibers.
\end{abstract}

Key words: enzyme activity, single muscle fiber, fiber type, suspension hypokinesia.

It has been reported that space flight causes an atrophy in the skeletal muscle (LeONARD et al., 1983). A similar phenomenon can be caused by the hindlimb suspension of rats in weightlessness simulation models (MUSACCHIA et al., 1980).

Received for publication March 20, 1989

* To whom correspondence should be addressed. 
Such atrophy is accompanied by a decrease in mitochondrial, but not in cytoplasmic, enzymes activity (OHIRA and Shimamura, 1987). Templeton et al. (1988) reported that following a 4-week suspension of rat hindlimbs, the percent distribution of type I fiber was reduced and that of type IIa fiber was significantly increased. On the other hand, RoY et al. (1987) reported that no significant changes in fiber-type distribution were observed in either the superficial region of the gastrocnemius or the tibialis anterior muscles following a 4-week hindlimb suspension in rats. These reports suggested that hindlimb suspension affected not only the functional profiles but also the structural profiles in the skeletal muscle fiber, however, skeletal muscle consists of three major types of fiber classified by contraction speed and metabolic profiles (PETER et al., 1972). Currently, it is not clear whether the structural and metabolic changes occurred equally or not in different types of fibers following hindlimb suspension. Roy et al. (1987) and HAuSCHKA et al. (1987) analyzed the size and metabolic properties of fast-twitch or slow-twitch fibers, respectively, following a 4-week hindlimb suspension in rats. They reported that the hindlimb suspension affected each type of muscle fiber differently, although the relationship between metabolic and ultrastructural profiles in each type of single muscle fiber was not examined.

In the present study, we examined the changes in ultastructural and metabolic profiles in single muscle fiber of different types following hindlimb suspesnion for 2 weeks in rats, using biochemical and electron microscopical techniques.

\section{MATERIALS AND METHODS}

Animal care and suspension procedure. Fourteen male Wistar strain rats (10 weeks old, 200-220 g) were used in the present study. They were randomly divided into two groups of 7 each: 1) control in cage (CON), and 2) hindlimb-suspended (SUS). All rats were maintained in a $12: 12 \mathrm{~h}$ light-dark cycle, with an ambient temperature of $23 \pm 1{ }^{\circ} \mathrm{C}$, and were given food and water ad libitum throughout the experiment. These studies were approved by the Animal Use Committee of the National Institute of Fitness and Sports (NIFS) and followed the American Physiological Society Animal Care Guidelines. Body suspension was performed by using the modified method reported by MusaCCHIA et al. (1980). The head was lowered about $10 \%$ to shift the fluid toward the head as in the weightless environment (HARGENs et al., 1983). The suspended rats were able to use forelimbs to move around a pivot, permitting access to food and water freely. Apparent changes in the structure and function of muscle fibers after 2-week suspension have been reported (DESPLANCHES et al., 1987; ElDER and McComas, 1987). Therefore, we decided that a period of 2-week suspension hypokinesia in this study would be appropriate. After a 2-week suspension, all rats were anesthetized with sodium pentobarbital $(5 \mathrm{mg} / 100 \mathrm{~g}$ body weight, i.p.); extensor digitorum longus (EDL) and soleus (SOL) muscles were removed from both hindlimbs and weighed as soon as possible before preparation for each analysis. Analysis of whole muscle homogenates 
of enzyme activities was performed by using left SOL and EDL muscles, and dissection of single muscle fibers was performed by using right SOL and EDL muscles, respectively.

Whole muscle enzyme activity. A portion of EDL and SOL was fully homogenized in ice-cold Tris- $\mathrm{HCl}$ buffer $(1.5 \mathrm{M}, \mathrm{pH} 7.0)$ for measurements of lactate dehydrogenase (LDH, EC: 1.1.1.27) and malate dehydrogenase (MDH, EC: 1.1.1.37) according to SUOMINEN and HEIKKINEN (1975). The remaining portion was separated into two and homogenized either in ice-cold triethanolamine buffer (50 mM, pH 7.6) containing $6 \mathrm{mM} \mathrm{Na}_{2} \mathrm{H}_{2}$ EDTA, or in ice-cold phosphate buffer (100 mM, pH 7.4) for measurement of phosphofructokinase (PFK, EC: 2.7.1.56) according to the method of SHONK and BOXER (1950) and succinate dehydrogenase (SDH, EC: 1.3.99.1) according to the method of CoOPERSTEIN et al. (1950), respectively. All enzyme activities were expressed in international units (I.U.)/mg protein. The total protein content of each homogenate was measured by the method of GOLDBERG (1973).

Enzyme activity in single muscle fiber. Single muscle fibers were dissected from both SOL and EDL muscles in ice-cold relaxing solution for mammals $(120 \mathrm{~mm}$ $\mathrm{KCl}, 0.5 \mathrm{~mm}$ EGTA, $4 \mathrm{~mm} \mathrm{MgCl}_{2}$, $10 \mathrm{~mm}$ PIPES, $10 \mathrm{~mm}$ ATP, pH 6.8). Several fragments were isolated from a fiber end, and torn apart longitudinally. Then they were placed on a cover glass with the intact cell membrane facing the glass surface, and stained histochemically (myofibrillar actomyosin ATPase, pre-incubation $\mathrm{pH}$ 4.3 or 10.4 (Guth and Samaha, 1970), and SDH (Nachlas et al., 1957)) in order to classify the fiber type into three classes: 1) slow-twitch oxidative (SO); 2) fast-twitch oxidative glycolytic (FOG); or 3) fast-twitch glycolytic (FG) fiber according to the method of PETER et al. (1972). SO fibers were mainly dissected from the SOL muscle, and FOG and FG fibers were mainly dissected from EDL muscles. After classifying the fiber type, the remaining parts of each fiber were used for measurement of enzyme activities, relative mitochondrial volumes, and lipid droplet volumes. For the analysis of the above-mentioned enzyme activity, each type of single muscle fiber was homogenized using a micro-glass homogenizer (TAKEKURA and Yoshioka, 1987).

Electron microscopical analysis. For analysis of relative mitochondrial and lipid droplet volumes using electron microscopy, the fragment of each type of single muscle fiber was fixed with $2.5 \%$ glutaraldehyde (GA) in cold Ringer's solution for 30-60 min and then transferred to the fixing solution consisting of $2.5 \%$ GA in $0.1 \mathrm{M}$ phosphate buffer. The fragments were post-fixed with $1 \%$ osmium tetroxide buffered with $100 \mathrm{~mm}$ phosphate. The fixation was processed at $4^{\circ} \mathrm{C}$. Each fragment was then dehydrated with graded ethanol and embedded in Epon resin. The longitudinal and transverse sections obtained by the LKB-microtome were mounted on the \#200 copper grids and were observed by a JEOL-100C electron microscope (Nihon Denshi Co. Ltd., Tokyo, Japan) with the calibration grid (No. 6002, Ernest F. Fullan Inc., New York, U.S.A.), and photographed. Stereological analysis of the volume of mitochondria and lipid droplet for each type of fiber was performed 
on the electron micrographs (WEIBEL, 1969).

Statistical analysis. The significance of differences between the cage-control (CON) and hindlimb-suspended (SUS) muscles or fibers was evaluated by Student's paired $t$-test. In all cases, the level of significance was set at $p<0.05$.

\section{RESULTS}

\section{Body weight and muscle wet weight}

At the end of the suspension, the mean body weights of the CON and SUS groups were approximately the same, i.e., $240.8 \pm 19.7$ and $221.5 \pm 34.6 \mathrm{~g}$, respectively. Following a 2 -week suspension, the mean body weight tended to reduce. However, the mean wet weight of the SOL muscle in the SUS group was $40 \%$ smaller than the CON group with a significant difference of $p<0.01$ order, but the mean wet weight of EDL was approximately the same in the two groups. Relative muslce weight of both muscles (muscle wet weight/body weight) also showed a similar tendency (Table 1).

\section{Whole muscle homogenate enzymes activity}

Glycolytic (LDH and PFK) and oxidative (SDH and MDH) enzyme activity in whole SOL and EDL muscle homogenates are shown in Table 2. SOL muscle mainly consists of slow-twitch fiber (approximately 95\%), and EDL muscle mainly consists of fast-twitch fiber (approximately $\sim 90 \%$ ), respectively (ARMSTRONG and PHeLPS, 1984). Consequently, glycolytic enzyme activity was higher in EDL muscle than SOL muscle, and oxidative enzyme activity was higher in SOL muscle than in EDL muscle of cage-control rats. There was no significant difference in LDH and SDH activity of both SOL and EDL muscles between the CON and SUS

Table 1. Body weight, muscle wet weight, and relative weight of SOL and EDL muscles in cage-control (CON) and hindlimb-suspended (SUS) rats.

\begin{tabular}{|c|c|c|c|c|c|c|c|c|}
\hline \multirow{2}{*}{ Group } & \multirow{2}{*}{$N$} & & \multicolumn{2}{|c|}{ Body weight (BW, g) } & \multicolumn{2}{|c|}{ Muscle weight (MW, mg) } & \multicolumn{2}{|c|}{$\mathrm{MW} / \mathrm{BW} \times 10^{4}$} \\
\hline & & & B.S. & A.S. & SOL & EDL & SOL & EDL \\
\hline $\begin{array}{r}\text { Control } \\
(\mathrm{CON})\end{array}$ & 7 & $\begin{array}{c}\text { M } \\
\text { S.D. }\end{array}$ & $\begin{array}{r}227.8 \\
17.8\end{array}$ & $\begin{array}{r}240.8 \\
19.7\end{array}$ & $\begin{array}{c}91.5 \\
9.87\end{array}$ & $\begin{array}{l}98.8 \\
15.4\end{array}$ & $\begin{array}{l}3.8 \\
0.27\end{array}$ & $\begin{array}{l}4.1 \\
0.1\end{array}$ \\
\hline $\begin{array}{l}\text { Suspended } \\
\text { (SUS) }\end{array}$ & 7 & $\begin{array}{c}\text { M } \\
\text { S.D. }\end{array}$ & $\begin{array}{r}236.0 \\
35.0\end{array}$ & $\begin{array}{r}221.5 \\
34.6\end{array}$ & $\begin{array}{l}54.7 \\
19.1\end{array}$ & $\begin{array}{r}101.0 \\
23.9\end{array}$ & $\begin{array}{l}2.4 \\
0.6\end{array}$ & $\begin{array}{l}4.5 \\
0.5\end{array}$ \\
\hline
\end{tabular}

Abbreivations: SOL, soleus muscle; EDL, extensor digitorum longus muscle; B.S., before suspension; A.S., after suspension. $* *$ Significant level, $p<0.01$. 
Table 2. Glycolytic and oxidative enzyme activities in SOL and EDL muscles in cage-control (CON) and hindlimb-suspended (SUS) rats.

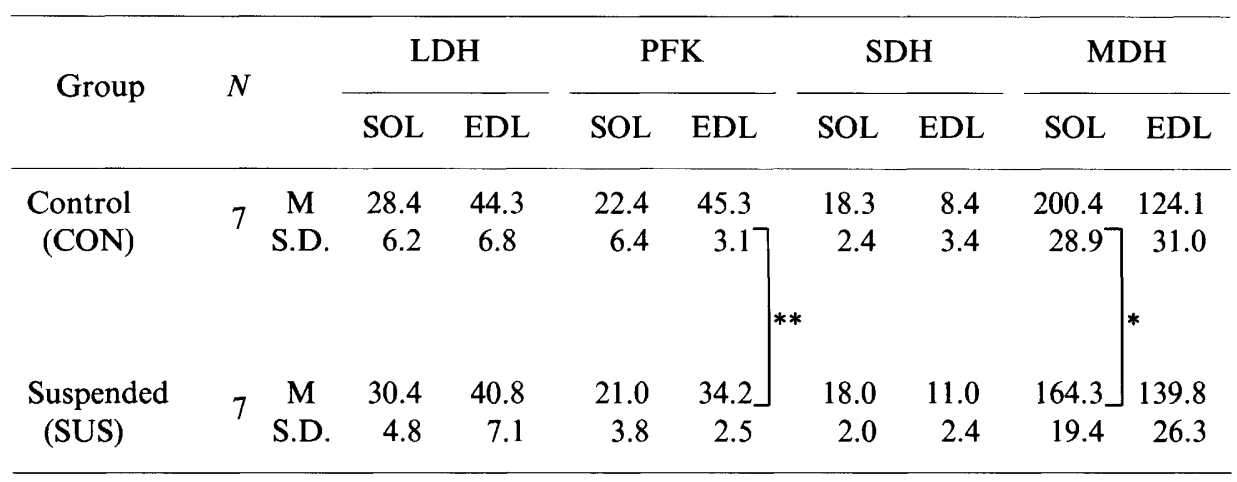

Abbreviations: SOL, soleus muscle; EDL, extensor digitorum longus muscle; LDH, lactate dehydrogenase; PFK, phosphofructokinase; SDH, succinate dehydrogenase; $\mathrm{MDH}$, malate dehydrogenase. All enzymes activities were expressed by international units (I.U.)/mg protein. Significant levels: $* p<0.05, * * p<0.01$.
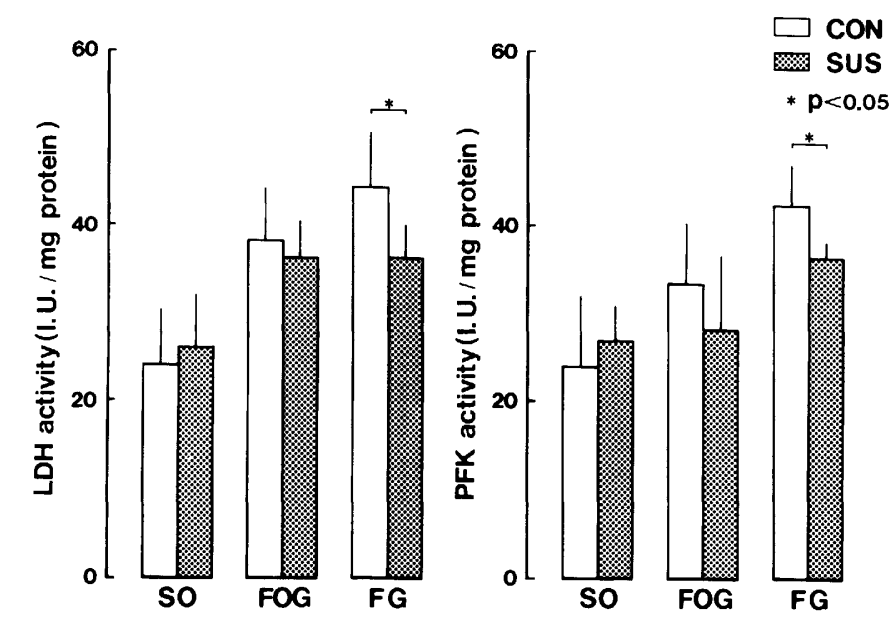

Fig. 1. Glycolytic (LDH and PFK) enzyme activities of different types of single muscle fibers in cage-control (CON) and hindlimb-suspended (SUS) rats. Abbreviations: LDH, lactate dehydrogenase; PFK, phosphofructokinase; SO, slow-twitch oxidative fiber; FOG, fast-twitch oxidative glycolytic fiber; FG, fast-twitch glycolytic fiber. Values are means with standard deviations.

groups. However, PFK activity of EDL muscle and MDH activity of SOL muscle in the SUS group was significantly (PFK, $p<0.01$; $\mathrm{MDH}, p<0.05)$ lower than that of the CON group. 
Single muscle fiber enzyme activity

Glycolytic (LDH and PFK) enzyme activity in each type of single muscle fibers of both the CON and SUS groups is shown in Fig. 1. Except for SO fiber, both LDH and PFK activities in the CON group were higher than those of the SUS

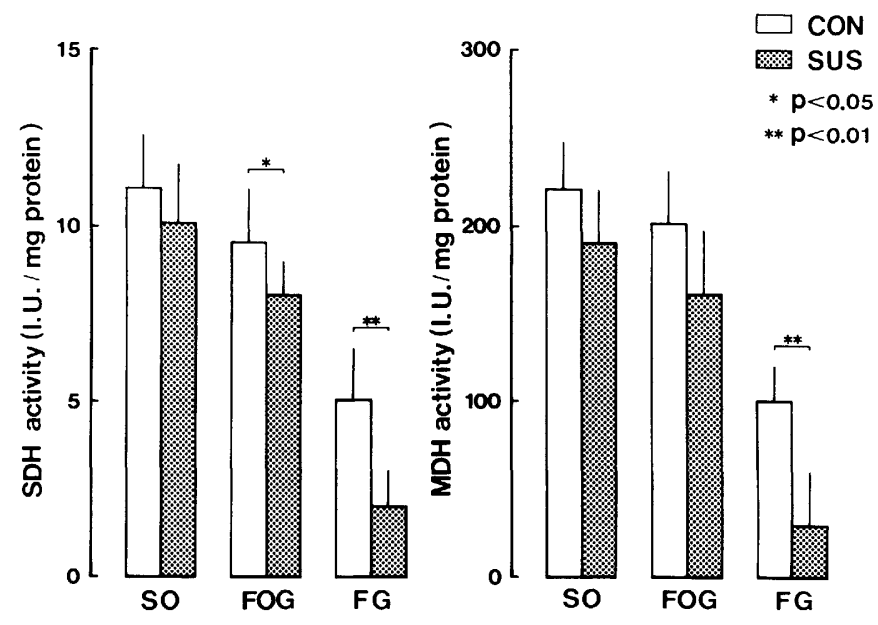

Fig. 2. Oxidative (SDH and $\mathrm{MDH}$ ) enzyme activities of different types of single muscle fibers in cage-control (CON) and hindlimb-suspended (SUS) rats. Abbreviations: $\mathrm{SDH}$, succinate dehydrogenase; $\mathrm{MDH}$, malate dehydrogenase; others are the same as in Fig. 1. Values are means with standard deviations.

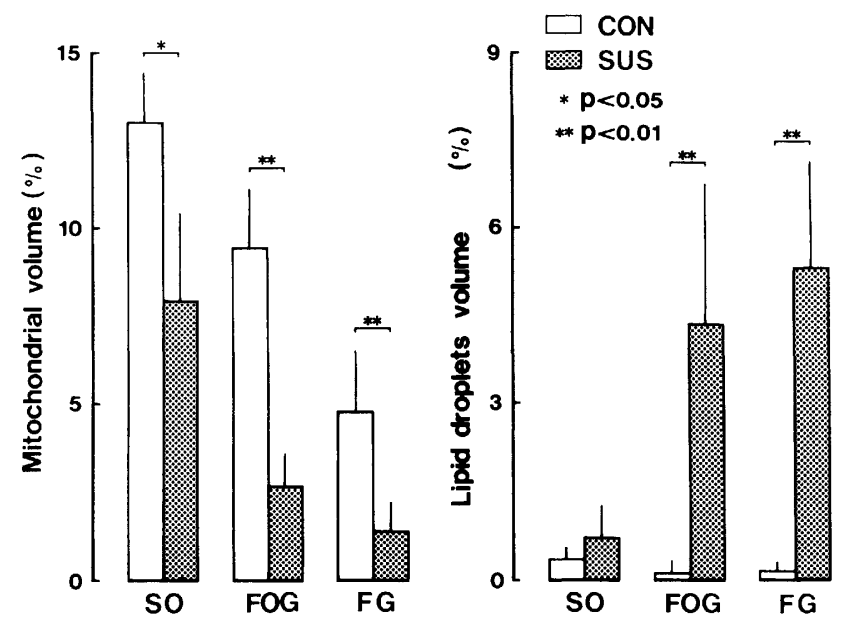

Fig. 3. Ultrastructural parameters (relative mitochondrial and lipid droplet volumes) of different types of single muscle fibers in cage-control (CON) and hindlimbsuspended (SUS) rats. Abbreviations are the same as in Fig. 1. Values are means with standard deviations. 
group. In SO fiber, both enzyme activities were higher in the SUS group than those of the CON group. Oxidative (SDH and $\mathrm{MDH}$ ) enzyme activities in each type of single muscle fibers are shown in Fig. 2. Oxidative enzyme activities in all types of single muscle fibers were higher in the CON group than those of the SUS group, with a significant difference of $p<0.05$ (SDH activity in FOG fiber) and $p<0.01$ (SDH and $\mathrm{MDH}$ activities in FG fiber), respectively. The decrease of oxidative enzyme activities following hindlimb suspension was remarkable in FG fiber.

\section{Ultrastructural parameters}

Relative volumes of mitochondria and lipid droplet analyzed stereologically from electron micrographs in each type of single muscle fiber of both groups are shown in Fig. 3. Relative volumes of mitochondria in all types of single muscle fibers of the CON group were significantly higher (SO, $p<0.05$; FG and FOG, $p<0.01)$ than those of the SUS group. On the other hand, relative lipid droplet volumes in all types of single muscle fibers in the SUS group were higher than those of the CON group, with a significant difference of $p<0.01$ (FG and FOG fibers).

Relationship between oxidative enzymes activity and relative mitochondrial volumes

Relationships between SDH or MDH activity and relative mitochondrial volumes in each type of single muscle fiber of both groups are shown in Figs. 4 and 5 , respectively. In general, oxidative enzyme activities depended on the

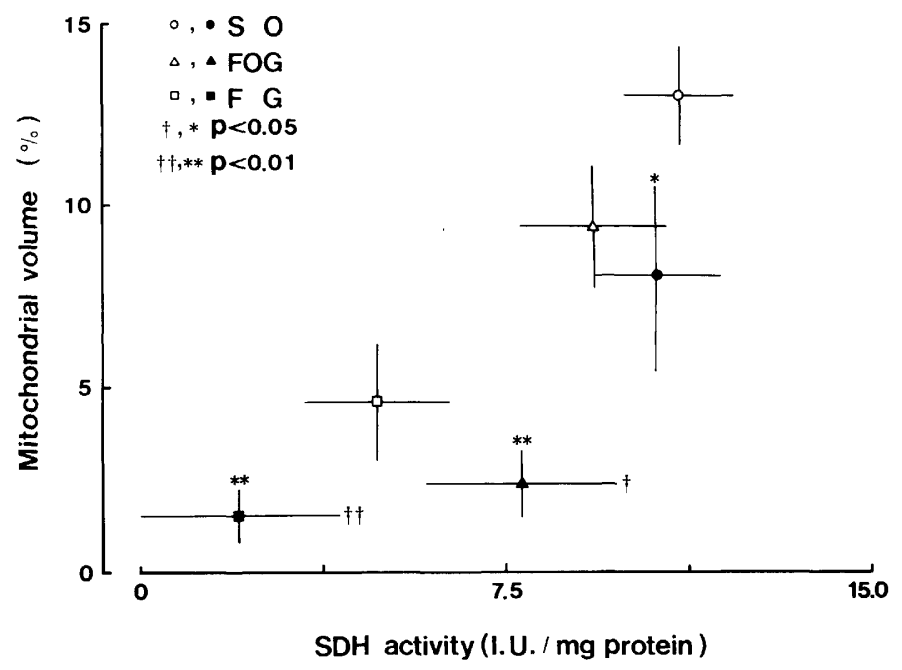

Fig. 4. Relationships between SDH activity and relative mitochondrial volumes of different types of single muscle fibers in cage-control (open marks) and hindlimbsuspended (closed marks) rats. Values are means with standard deviations. Signifcant levels are expressed between cage-control and hindlimb-suspended rats in the same type of fibers $\left(*\right.$ mitochondrial volume; ${ }^{\dagger} \mathrm{SDH}$ activity). Abbreviations are the same as in Fig. 2. 


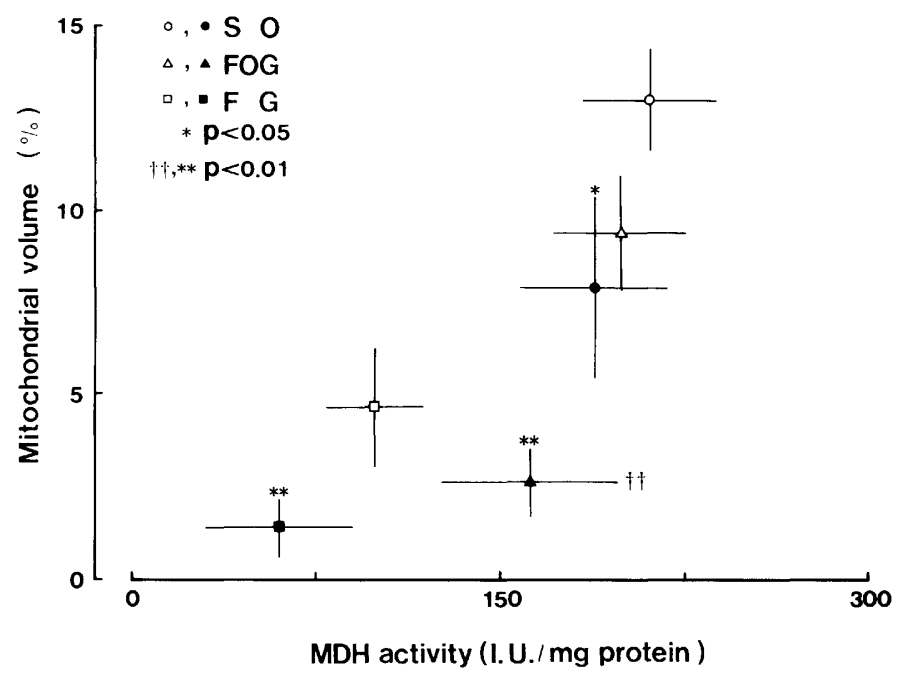

Fig. 5. Relationships between MDH activity and relative mitochondrial volumes of different types of single muscle fibers in cage-control (open marks) and hindlimbsuspended (closed marks) rats. Values are means with standard deviations. Significant levels are expressed between cage-control and hindlimb-suspended rats in the same type of fibers $\left({ }^{*}\right.$ mitochondrial volume; ${ }^{\dagger} \mathrm{MDH}$ activity). Abbreviations are the same as in Fig. 2.

mitochondrial volume. Relative mitochondrial volumes in all types of single muscle fiber of the CON group were significantly higher than those of the SUS group. However, SDH and MDH activities in each type of single muscle fiber did not show similar tendencies. The relative mitochondrial volume in SO fiber of the SUS was significantly $(p<0.05)$ lower than that of the CON group. However, SDH and $\mathrm{MDH}$ activities in SO fibres were not different between the CON and SUS groups. The same tendency was also observed in FG fiber.

\section{DISCUSSION}

Wet weight and relative weight of SOL muscle in the SUS group were significantly lower than those of the CON group. But in EDL muscle, no significant decrease of the wet weight and relative weight were observed following suspension. These results suggested that hypokinesia through suspension caused an atrophy mainly of slow-twitch fibers as was reported previously (ILYVA-KAKUEVA et al., 1976). It was reported that whole-body suspension is characterized by a different atrophy of hindlimb muscles (SOL $>$ gastrocnemius $>$ plantaris $=E D L)$ and increased excretion of nitrogenous products (MUSACCHIA et al., 1980, 1983). WALMSLEY et al. (1978) reported that maintenance of body weight was mainly controlled by the S-type motor unit. Since SOL mainly consists of the S-type motor units (BURKE 
and EDGERTON, 1975), significant atrophy may be observed mainly in SOL muscle following suspension. We concluded that the degree of skeletal muscle atrophy following body or hindlimb suspension depended on the number of S-type motor units in the skeletal muscle.

Glycolytic and oxidative enzyme activity of whole SOL and EDL muscle homogenates decreased except for LDH activity in SOL muscle and SDH activity in EDL muscle following hindlimb suspension. SIMARD et al. (1985) reported that glycolytic and oxidative enzyme activity decreased in both SOL and gastrocnemius muscles following a 3-week suspension hypokinesia. They also reported that these changes might reflect a transformation of fast-twitch fibers to slow-twitch fibers. On the other hand, Templeton et al. (1984) and THOMAsON et al. (1987) reported an increase of fast-type myosin isozyme composition and fast-twitch fiber number, but no change in the total fiber number in various muscles following suspension hypokinesia. These results suggest that transformation from slow-twitch to fast-twitch type fiber occurred following suspension. At present, there is no consensus on the muscle fiber transformation following the hindlimb suspension.

Glycolytic enzyme activity in fast-twitch (FG and FOG) fiber and oxidative enzyme activity in SO fiber decreased following hindlimb suspension, respectively. Decrease of oxidative enzyme activities was remarkable in fast-twitch (FG and FOG) fibers. Decrease of glycolytic and oxidative enzyme activity in both SOL and EDL whole homogenates depended on the decrease of these enzyme activities in each fiber, but not on fiber-type transformation. These results suggested that the muscle contractile energy metabolism decreased reflecting hypokinesia due to the suspension. We cannot explain why these results were mainly observed in fast-twitch fiber. As mentioned above, maintenance of the body weight mainly depends on the slow-type motor units in the skeletal muscle. Therefore, suspension disuse mainly affected the slow-twitch fiber. It was reported that the contraction time and half-relaxation time of skeletal muscle decreased following hindlimb suspension (HERBERT et al., 1988). These results reflected the increase of fast-type myosin isozyme composition (TEMPLETON et al., 1988). If fiber-type transformation from slow-twitch to fast-twitch fibers were to occur following hindlimb suspension, the decrease of glycolytic enzyme in fast-twitch fiber is a contradictory finding.

Relative mitochondrial volumes in all types of muscle fibers of the SUS group were significantly lower than those of the CON group. There were no significant differences in the rate of decrease of relative mitochondrial volume in three types of single muscle fibers. Many factors control the mitochondrial volume, e.g. hypoxia (Pelosi and Agliati, 1968), anoxia (Oudea, 1963), temperature (Brooks et al., 1971), pH (CEREIJO-Santalo, 1966), DNA content (LAugens and Gonez-Dumm, 1968), elemental composition (LEHNINGER, 1962), etc. Consequently, mitochondria is an organelle which is highly sensitive to various physiological stimuli. It was not clear whether these factors changed in response to hindlimb suspension in each type of fiber. It was reported that absolute DNA levels did not change in atrophic 
muscle following hindlimb suspension (STEFFEN and MUSACCHIA, 1984). It was not clear whether mitochondrial DNA levels changed or not, so further studies are necessary to clarify the decrease of relative mitochondrial volume in all types of muscle fibers following hindlimb suspension (YosHioka et al., 1988).

Relative lipid droplet volume in each type of fiber in the SUS group was higher than those of the CON group. In particular, there were significant differences in FG and FOG fibers between the CON and SUS groups. Oxidative enzyme activity in all types of fiber decreased following hindlimb suspension. It was not clear in the present study whether fatty oxidative enzyme activity decreased or not in each type of fiber in response to hindlimb suspension. However, these results reflected the decrease of oxidative capacity of lipid following hindlimb suspension in all types of fibers (YoshioKa et al., 1988).

Relative mitochondrial volumes in all types of fibers of the SUS group were lower than those of the CON group; however, oxidative enzyme activity did not show the same tendency following a 2-week hindlimb suspension. If the relative volumes analyzed reflect directly structural alterations in mitochondria, oxidative enzyme activity depended on mitochondrial volume and number. In this report, we did not present changes in mitochondrial volume induced by structural alterations. However, changing patterns in oxidative capacity and mitochondrial volume in response to hindlimb suspension differed in each type of muscle fiber. Significant differences were observed in all types of fibers in terms of relative mitochondrial volume, but were not observed in oxidative enzyme activity in the CON and SUS groups. These results suggested that the changes in oxidative capacity in response to hindlimb suspension did not completely depend on mitochondrial alterations.

In conclusion, changes in the structural and functional profiles of each type of single muscle fibers following hindlimb suspension were influenced by different factors, and structural changes mainly appeared in slow-twitch fibers and functional changes mainly appeared in fast-twitch fibers, respectively, following hindlimb suspension.

We thank Associate Professor S. H. Shang of the National Institute of Fitness and Sports and J. Patrick Barron of St. Marianna University School of Medicine for their reviews of the English manuscript.

\section{REFERENCES}

Armstrong, R. B. and Phelps, R. O. (1984) Muscle fiber type composition of rat hindlimb. Am. J. Anat., 171: 259-272.

Brooks, G. A., Hittleman, K. J., Faulkner, J. A., and Beyer, R. E. (1971) Temperature, skeletal muscle mitochondria function changes and oxygen debt. J. Physiol. (Lond.), 220: $1053-1059$.

Burke, R. E. and Edgerton, V. R. (1975) Motor unit properties and selective involvement. Exerc. Sport Sci. Rev., 3: 31-81. 
Cereijo-Santalo, R. (1966) Mitochondrial swelling at acid pH. Can. J. Biochem., 44: 695-706.

Cooperstein, S. T., LAZOrow, A., and Kurfess, N. J. (1950) A spectrophotometric method for determination of succinate dehydrogenase. J. Biol. Chem., 186: 129-139.

Desplanches, D., Mayet, M. H., Sempore, B., and Flandrois, R. (1987) Structural and functional responses to prolonged hindlimb suspension in rat muscle. J. Appl. Physiol., 63: $558-563$.

Elder, G. C. B. and McComas, A. J. (1987) Development of rat muscle during short- and long-term hindlimb suspension. J. Appl. Physiol., 62: 1917-1923.

GoldBERG, M. L. (1973) Quantitative assay for submicrogram amounts of protein. Anal. Biochem., 51: 240-246.

Guth, L. and Samaha, F. J. (1970) Procedure for the histochemical demonstration of actomyosin ATPase. Exp. Neurol., 28: 365-367.

Hargens, A. R., Tipton, C. M., Gollnick, P. D., Mubarak, S. J., Tucker, B. J., and AKeson, W. H. (1983) Fluid shifts and muscle function in humans during acute simulated weightlessness. J. Appl. Physiol., 54: 1003-1009.

HauschKa, E. O., Roy, R. R., and Edgerton, V. R. (1987) Size and metabolic properties of single muscle fibers in rat soleus after hindlimb suspension. J. Appl. Physiol., 62: 2338-2347.

HerberT, M. E., Roy, R. R., and Edgerton, V. R. (1988) Influence of one-week hindlimb suspension and intermittent high load exercise on rat muscles. Exp. Neurol., 102: 190-198.

Ilyva-Kakueva, E. I., Portugalov, V. V., and Krivenkova, N. P. (1976) Space flight effects on the skletal muscles of rats. Aviat. Space Environ. Med., 47: 700-703.

Laugens, E. P. and Gonez-Dumm, C. L. (1968) Deoxyribonucleic acid synthesis in rat heart mitochondria after acute and exhaustive exercise. Experientia, 24: 163-164.

LeHNINGER, A. L. (1962) $\mathrm{H}_{2} \mathrm{O}$ uptake and extrusion by mitochondria in relation to oxidative phosphorylation. Physiol. Rev., 42: 467-517.

Leonard, J. J., Leach, C. S., and Rambaut, P. C. (1983) Quantitation of tissue loss during prolonged space flight. Am. J. Clin. Nutr., 38: 666-679.

Musacchia, X. J., Deavers, D. R., Meininger, G. A., and Davis, T. P. (1980) A model for hypokinesia: Effects on muscle atrophy in rat. J. Appl. Physiol., 48: 479-486.

Musacchia, X. J., StefFen, J. M., and Deavers, D. R. (1983) Rat hindlimb muscle responses to suspension hypokinesia/hypodynamia. Aviat. Space Environ. Med., 54: 1015-1020.

Nachlas, M. M., Tsou, K. C., Souza, E. D., Cheng, C. S., and Seligman, A. M. (1957) Cytochemical demonstration of succinate dehydrogenase by use of a new p-nitrophenyl substitute diterazole. J. Histochem. Cytochem., 5: 420-436.

OHira, Y. and Shimamura, H. (1987) Effects of electrical stimulation on muscle mass and metabolic characteristics in suspended rats. Proc. Nihon Univ. Symp. Aerosp. Sci., 1: $71-72$.

OUDEA, P. R. (1963) Anoxic changes of liver cells. Electron microscope study after injection of celloidal mercury. Lab. Invest., 12: 386-394.

Pelosi, C. and Agliati, C. (1968) The heart muscle in functional overloaded and hypoxia. Lab. Invest., 18: 86-93.

Peter, J. B., Barnard, R. J., Edgerton, V. R., Gillespie, C. A., and Stempel, K. E. (1972) Metabolic profiles of three fiber types on the skeletal muscle in guinea pig and rabbits. Biochemistry, 11: 2627-2633.

Roy, R. R., Bello, M. A., Bouissou, P., and Edgerton, V. R. (1987) Size and metabolic properties of fibers in rat fast-twitch muscles after hindlimb suspension. J. Appl.

Vol. 39, No. 3, 1989 
Physiol., 62: 2348-2357.

Shonk, C. E. and Boxer, G. E. (1950) Enzyme patterns in human tissues. I. Methods of the determination of glycolytic enzymes. Cancer Res., 24: 709-721.

Simard, C., LaCaille, M., and Vallieres, J. (1985) Enzymatic adaptations to suspension hypokinesia in skeletal muscle of young and old rats. Mech. Aging Dev., 33: 1-9.

StefFen, J. M. and Musacchia, X. J. (1984) Effect of hypokinesia and hypodynamia on protein, RNA, and DNA in rat hindlimb muscles. Am. J. Physiol., 247: R728-R732.

Suominen, H. and Heikrinen, E. (1975) Enzyme activities in muscle and connective tissue of M. Vastus Lateralis in habitually trained and sedentary 33 to 70 -year-old men. Eur. J. Appl. Physiol., 34: 249-254.

Takekura, H. and Yoshioka, T. (1987) Determination of metabolic profiles on single muscle fibres of different types. J. Muscle Res. Cell Motil., 8, 342-348.

Templeton, G. H., Padalino, M., Manton, J., Glasberg, M., Silver, C. J., Silver, P., Demartino, G., Lecony, T., Klug, G., Hagler, H., and Sutko, J. L. (1984) Influence of suspension hypokinesia on rat soleus muscle. J. Appl. Physiol., 56: 278-286.

Templeton, G. H., Sweeney, H. L., Timson, B. F., Padalino, M., and Dudenhoeffer, G. A. (1988) Changes in fiber composition of soleus muscle during hindlimb suspension. J. Appl. Physiol., 65: 1191-1195.

Thomason, D. B., Herrick, R. E., Surdyka, D., and Baldwin, K. M. (1987) Time course of soleus muscle myosin expression during hindlimb suspension and recovery. J. Appl. Physiol., 63: 130-137.

Walmsley, B., Hodgson, J. A., and Burke, R. E. (1978) The forces produced by medial gastrocnemius and soleus muscle during locomotion in free moving cats. $J$. Neurophysiol., 41: 1203-1216.

WeIBeL, E. R. (1969) Stereological principles for morphometry in electron microscopic cytology. Int. Rev. Cytol., 26: 235-302.

Yoshioka, T., Takekura, H., OhiRa, Y., and Saiki, H. (1988) The mitochondrial volume and fiber type transition of skeletal muscle after suspension hypokinesis in rat. Jpn. J. Aerospace. Environ. Med., 25: 87-96 (in Japanese). 